\title{
Ocular changes with oral and transepidermal diethylcarbamazine therapy of onchocerciasis
}

\author{
HUGH R. TAYLOR ${ }^{1}$ AND BRUCE M. GREENE ${ }^{2}$ \\ From the ${ }^{1}$ International Center for Epidemiologic and Preventive Ophthalmology, Wilmer Ophthalmological \\ Institute, Johns Hopkins Medical Institutions, Baltimore, Maryland 21205, USA, and the \\ ${ }^{2}$ Division of Geographic Medicine, Department of Medicine, Case Western Reserve University and \\ University Hospitals, Cleveland, Ohio 44106, USA
}

SUMMARY Twenty men with moderate infection of Onchocerca volvulus were studied in a doublemasked, controlled clinical trial to compare the safety and efficacy of oral diethylcarbamazine (DEC) with topical DEC lotion. Visual acuity and colour vision did not alter during the 6 months of observation, although 2 patients receiving DEC lotion and 3 patients receiving oral DEC developed either visual field constriction or optic atrophy. Fluffy corneal opacities were common in both groups. Intraocular microfilariae also appeared in both groups but to a greater extent in those receiving DEC lotion. New chorioretinal changes developed in 4 men receiving lotion and in only 1 receiving tablets. It is concluded that DEC lotion offers no advantage over tablets in the treatment of ocular onchocerciasis and in fact may be associated with more ocular complications than the conventional oral treatment.

Onchocerciasis, or river blindness, is the disease caused by the filarial parasite Onchocerca volvulus. It is characterised by the presence of relatively few adult worms encapsulated in subcutaneous nodules and invasion of the body, especially the skin and the eyes, by millions of microfilariae. The main disability caused by onchocerciasis is blindness, either from corneal or chorioretinal scarring, or from the sequelae of anterior uveitis. The pathological changes seen with onchocerciasis appear to be related directly or indirectly to the death of microfilariae.

While recent efforts have resulted in an improved understanding of the clinical presentation and natural history of onchocerciasis, methods of treating the disease remain unsatisfactory. Suramin sodium is the only clinically acceptable drug that kills the adult worms of Onchocerca volvulus. Its usefulness, however, is severely limited because it must be given intravenously, and it has a number of toxic side effects. ${ }^{1}$ Diethylcarbamazine (DEC), on the other hand, has relatively few intrinsic toxic side effects. ${ }^{2}$ But, although it promotes killing of microfilariae in vivo, it has no demonstrable effect on adult worms. ${ }^{3}$ In addition, as initially described by Mazzotti, ${ }^{4}$ DEC

Correspondence to Hugh R. Taylor, MD, Johns Hopkins Hospital, Wilmer Institute, Woods Research BIdg., Room 261, Baltimore, Maryland 21205, USA treatment of people with onchocerciasis frequently causes serious systemic, dermal, and ocular side effects.

Although DEC is readily absorbed when given by mouth, its transepidermal application also has been advocated. ${ }^{5}$ DEC citrate is absorbed by the skin, and in theory the transepidermal application should provide a therapeutic concentration of the drug in the skin, where most of the microfilariae reside, with minimal systemic absorption. It was hoped that a reduction in systemic absorption would lead to a decrease in systemic and ocular side effects.

A recently reported double-masked, controlled clinical trial evaluated the safety and efficacy of oral DEC and topical DEC in Liberian males with moderate infections of $O$. volvulus. ${ }^{3}$ This study showed that topical DEC was not only less effective than oral DEC in reducing the number of microfilariae in skin snips, but that topical DEC therapy was accompanied by at least as many systemic side effects as oral DEC, if not more. Despite this, it has been suggested that the use of topical DEC may have some role in the reduction of intraocular microfilariae. ${ }^{6}$

The purpose of this report is to present in detail the ocular changes observed in a double-masked controlled trial which compared oral and transepidermal administration of DEC. 


\section{Materials and methods}

\section{SUBJECTS}

Twenty male employees of the Firestone Plantation Company, Harbel, Liberia, were selected on the basis of their having a moderate infection with $O$. volvulus, as determined by skin-snip microfilariae count, without having significant visual impairment or other significant illness. The methodology used in this study has been described in detail elsewhere. ${ }^{3}$

\section{CLINICAL EXAMINATION}

A detailed ophthalmological examination was performed, including visual acuity, confrontation visual fields (with a $0.8 \mathrm{~cm}$ diameter red target), colour vision (AO pseudoisochromatic plates), and pupillary response. After head-down posturing, the cornea and anterior chamber were examined with a Haag-Streit Series 900 slit-lamp for the presence of microfilariae, corneal changes, and uveitis. Intraocular pressure was measured with a pneumatonometer. Mydriatic direct and indirect ophthalmoscopy were performed. Colour photographs of the optic disc and other fundal structures were taken with a Kowa fundus camera, and external ocular photographs were taken with a $35 \mathrm{~mm}$ camera with a macro lens.

Patients were admitted to hospital for the first 2 weeks of treatment. The ophthalmic and general physical examinations were performed before treatment and again at $1,3,7$, and 14 days, and at 2 and 6 months after the start of treatment. Skin snips also were taken at these times to determine the microfilarial density in the skin.
TREATMENT PROTOCOL

The patients were randomised into 2 groups in a double-masked fashion. One group, called the 'tablet group', received DEC citrate tablets $(50 \mathrm{mg}$ tablet, Lederle Laboratories, Valley Stream, NY) and placebo lotion (Nivea lotion, Biersdorf, South Norwalk, Conn.). The other group, called the 'lotion group', received a placebo tablet (Lederle), and 2\% DEC lotion made by adding powdered DEC citrate to the Nivea lotion to make a $2 \%(\mathrm{w} / \mathrm{v})$ preparation. On the first day of therapy all patients received 1 tablet, either DEC or placebo, and $15 \mathrm{ml}$ of lotion, either placebo or DEC. The lotion was applied to the whole body except to the scalp and the soles of the feet. For the next 7 days tablets were given 3 times daily and lotion once daily after a bath. From the second week patients were given tablets and lotion on only 1 day a week. During the first 2 months 3 tablets were taken in 3 divided doses on 1 day a week (total dose, $150 \mathrm{mg}$ ), and for the last 4 months 4 tablets were given in 2 divided doses (total dose, $200 \mathrm{mg}$ ). Medication was taken or applied under direct supervision.

\section{Results}

\section{GENERAL FEATURES}

Randomisation produced 2 groups that closely resembled each other. The men in the 2 groups were of similar age (mean age for the lotion group was $39 \cdot 8 \pm 11 \cdot 2$ years and for the tablet group $37 \cdot 4 \pm 14 \cdot 3$ years) and had comparable involvement with onchocerciasis (Table 1). All patients had palpable nodules. The geometric means of microfilarial counts

Table 1 Summary of important ocular changes seen in 20 men enrolled in a double-masked trial comparing DEC lotion with DEC tablets

\begin{tabular}{|c|c|c|c|c|c|c|c|c|}
\hline $\begin{array}{l}\text { Patient } \\
\text { number }\end{array}$ & $\begin{array}{l}\text { Pretreatment } \\
\text { sclerosing } \\
\text { keratitis }\end{array}$ & $\begin{array}{l}\text { Intraocular } \\
\text { mf at } 6 \text { months }\end{array}$ & $\begin{array}{l}\text { Fluffy corneal } \\
\text { opacities } \\
\text { at } 6 \text { months }\end{array}$ & $\begin{array}{l}\text { Pretreatment } \\
\text { chorioretinal } \\
\text { changes }\end{array}$ & $\begin{array}{l}\text { New } \\
\text { chorioretinal } \\
\text { changes }\end{array}$ & $\begin{array}{l}\text { Optic nerve } \\
\text { pallor }\end{array}$ & $\begin{array}{l}\text { Visual field } \\
\text { constriction }\end{array}$ & Other comments \\
\hline \multicolumn{9}{|c|}{ DEC lotion } \\
\hline 1 & ++ & + & + & + & & & & Penetrating injury, OD \\
\hline 2 & & & & + & & & & \\
\hline 6 & + & + & & + & + & & & \\
\hline 8 & ++ & + & + & & + & & & \\
\hline 10 & & + & + & + & + & + & & Colour blind (congenital) \\
\hline 11 & + & + & & & & & & \\
\hline 14 & & & & & & & & \\
\hline 16 & & & + & + & & + & + & Anisocoria, vitritis \\
\hline 17 & ++ & & & & + & & & \\
\hline 20 & & + & + & & & & & \\
\hline \multicolumn{9}{|c|}{ DEC tablets } \\
\hline 3 & & + & & + & + & & & \\
\hline 4 & + & & & + & & & & \\
\hline 5 & & & & + & & & & \\
\hline 7 & & & & & & + & & \\
\hline 9 & + & & & + & & & & \\
\hline 12 & ++ & & & & & & + & \\
\hline \multicolumn{9}{|l|}{13} \\
\hline \multirow{2}{*}{\multicolumn{9}{|c|}{18}} \\
\hline & & & & & & & & \\
\hline 19 & ++ & + & + & + & & & + & Limbitis \\
\hline
\end{tabular}


Fig. 1 The geometric means of the mean microfilarial count in skin snips from the 10 persons receiving $D E C$ tablets and the 10 persons receiving $D E C$ lotion for 6 months plotted on a semilog scale. Error bars indicate the standard error of the mean. For comparison, the pretreatment arithmetic mean ( \pm standard deviation) was $24 \cdot 3( \pm 10 \cdot 0)$ for the tablet group and $29 \cdot 8( \pm 16 \cdot 0)$ for the lotion group.

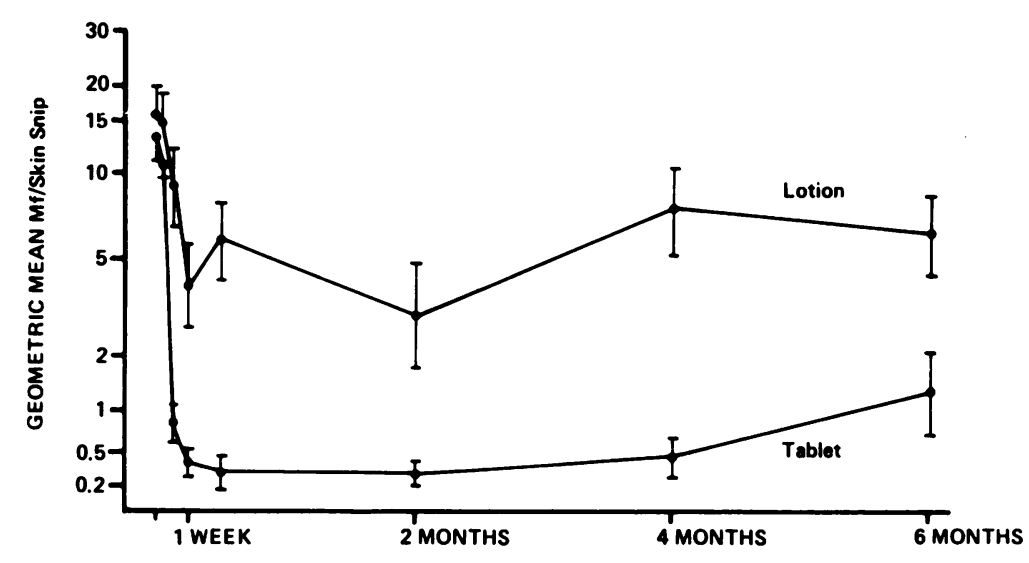

constriction of the superior field in the left eye was noticed at 14 days. At 2 months the fields were constricted to 20 degrees in the left eye and 5 to 10 degrees in the right. In the left eye the inferonasal quadrant seemed to be relatively spared. At 6 months there was generalised constriction to 10 to 15 degrees in each eye. In the third patient who received DEC lotion (patient 16) visual fields were noted to be full at the pretreatment examination and again at 2 weeks and at 2 months. However, at 6 months visual fields in both eyes were found to be constricted to 5 to 10 degrees.

\section{PUPILLARY RESPONSES}

Pupillary responses were normal in all patients before treatment with the exception of 1 man in the lotion group (patient 16 ) who had anisocoria with a mildly miotic right pupil. No change was observed in the pupillary reactions in this patient or in any other patient during this study.

\section{INTRAOCULAR MICROFILARIAE}

Before treatment microfilariae were not observed in the cornea or anterior chamber of any patient in either group. During the first 2 weeks of treatment microfilariae were seen commonly in the eyes of people in both groups (Fig. 2A). However, at both 2 and 6 months only 2 people receiving DEC tablets had intraocular microfilariae, while 6 people receiving DEC lotion did. During the 6 months of observation all 10 people receiving DEC lotion had intraocular microfilariae detected at some time, whereas only 7 of those receiving DEC tablets did. Five people receiving lotion had microfilariae on 4 or more of the 6 eye examinations, whereas only 2 people receiving tablets did: none had intraocular microfilariae before treatment. The mean number of microfilariae in the cornea and in the anterior chamber also was greater in those 

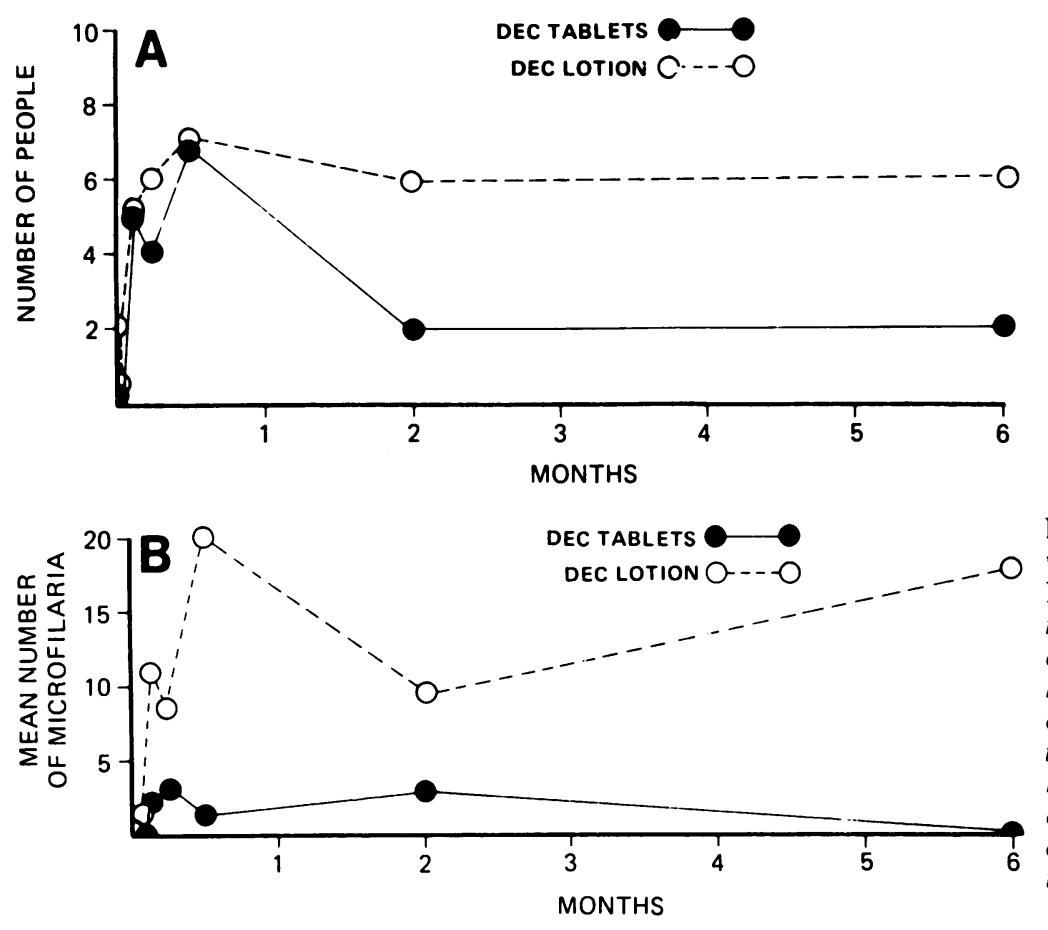

Fig. 2 A. The number of people with intraocular microfilariae. B. The mean number of microfilariae in the anterior chamber in those with intracameral microfilariae. C. The mean number of microfilariae in the cornea in those with intracorneal microfilariae. There were 10 men in each group, and they were examined during 6 months of treatment.

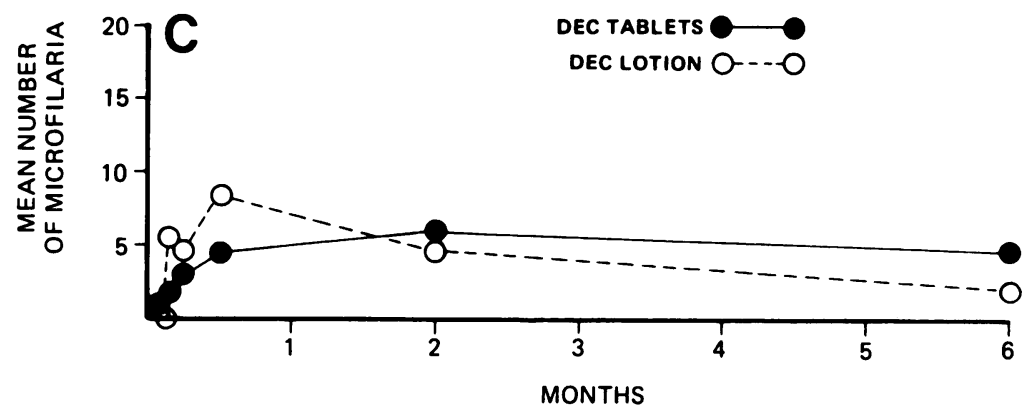

receiving DEC lotion than in those receiving tablets (Figs. 2B and C).

\section{KERATITIS}

Punctate keratitis (fluffy or snowflake corneal opacities) was present in 1 patient in each group before treatment. During treatment it appeared in a further 6 patients receiving DEC lotion and in 7 patients receiving DEC tablets. Most fluffy opacities were seen during the first 2 weeks (Fig. 3A). At 6 months, however, 5 patients receiving DEC lotion still had fluffy opacities, although they were seen in only 1 patient receiving DEC tablets at this time.

Before the start of treatment early sclerosing keratitis was present in 2 patients in the tablet group and 3 patients in the lotion group. An additional 3 patients in the tablet group and 2 patients in the lotion group had 'haze' in the peripheral cornea, which was most marked in the nasal and temporal areas in the interpalpebral fissure. This is regarded as the earliest slit-lamp sign of sclerosing keratitis. No change or progression of sclerosing keratitis was noticed during the study in men in either group.

\section{LIMBITIS}

One patient receiving DEC tablets (patient 19) developed severe limbitis and bulbar conjunctival chemosis on the day after starting oral DEC. This had almost entirely disappeared by 14 days. Despite careful examination the specific globular limbitis described as occurring during the treatment of onchocerciasis with DEC was not observed. ${ }^{7}$ 
Fig. 3 A. The number of people with fluffy corneal opacities. B. The number of people with anterior uveitis. There were 10 men in each group, and they were examined during 6 months of treatment.
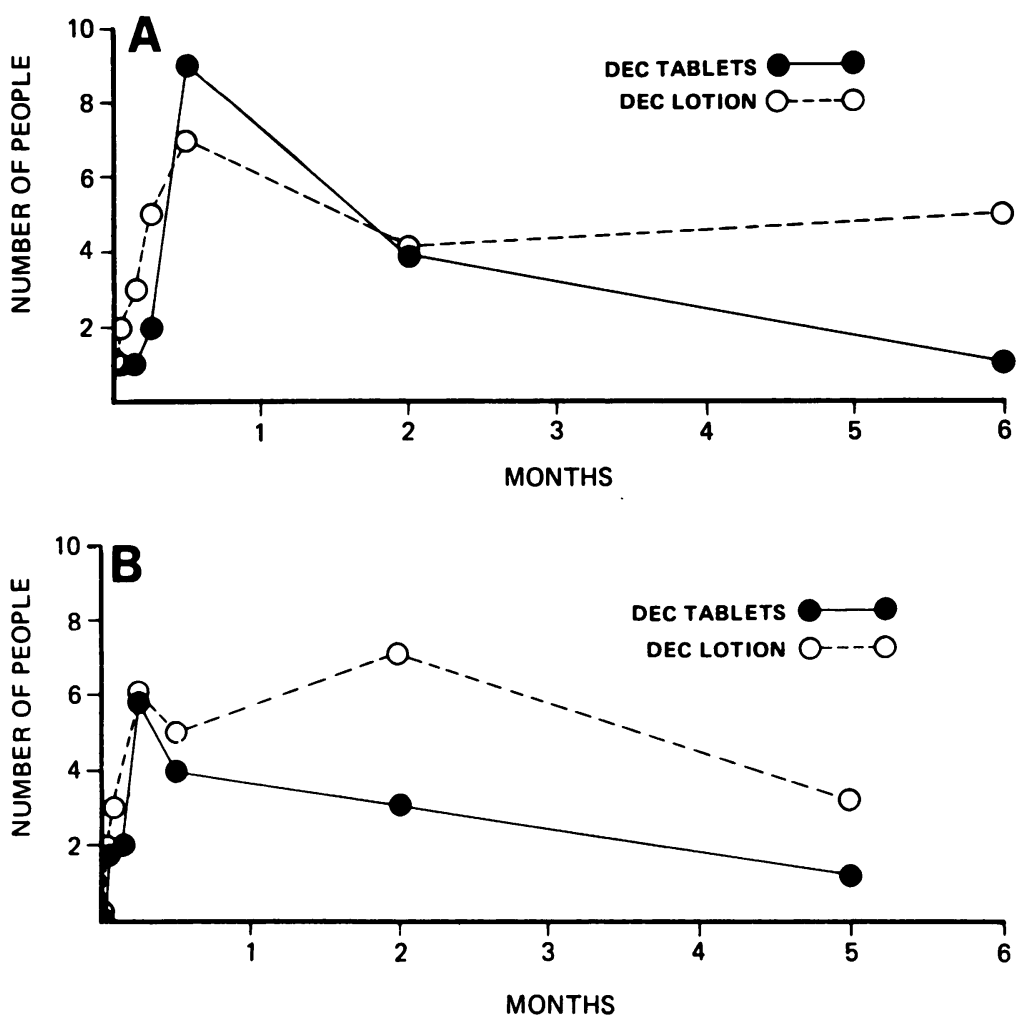

\section{ANTERIOR UVEITIS}

Anterior uveitis, as determined by the presence of cells or a flare in the anterior chamber, was not found in any patient before treatment. Within 7 days of the start of therapy, however, 6 patients in each group had developed signs of anterior uveitis. In only 1 patient in each group was this more severe than a mild uveitis (patients 15 and 16). After the first week of treatment anterior uveitis was seen in fewer people receiving oral DEC than in those receiving lotion, so that at 2 months only 3 of the tablet group had mild uveitis, whereas 7 patients receiving DEC lotion did (Fig. 3B).

\section{OPTIC DISC CHANGES}

Before the commencement of treatment the optic discs of all patients were considered normal. In 1 patient (patient 1) the optic disc of the right eye could not be examined because of the presence of a traumatic cataract. During the study definite changes were seen in the optic nerve of 2 men (both receiving DEC lotion), and questionable changes were seen in a third (treated with DEC tablets).

The patient receiving DEC tablets (patient 7) was thought to have questionable pallor of the optic nerve head of each eye at 6 months. No changes could be demonstrated on the stereo colour photographs, nor was there a change in his visual fields or colour vision. The cup:disc ratio remained at $0 \cdot 2$ on the right and $0 \cdot 3$ on the left throughout this study. One patient receiving DEC lotion (patient 10) had questionable pallor of his discs at 2 months, and at 6 months he had definite generalised pallor of both discs. Again no change could be detected in the photographs of the discs - in particular, a small pre-existing inferotemporal nerve fibre loss in the right eye remained unchanged during this period. Similarly, there was no change in his visual fields nor in the cup:disc ratio, which remained at 0.6 in both eyes. This patient had defective colour vision at the start of treatment, and this showed no change during the study. The other patient, who showed optic disc changes while being treated with DEC lotion (patient 16), also showed a generalised pallor of both discs. This was first noted at 6 months, although, again, no changes were detected in the photographs of the discs nor in the patient's colour vision. There was, however, marked visual field constriction. The visual fields in each eye, which had been full up to 2 months, were constricted to 5 to 10 degrees at 6 months when the disc pallor was seen. This patient had pre-existing anisocoria with miosis of the right pupil, and bilateral vitritis which became 
more severe during the study. The optic nerve of each eye showed no cupping during the period of observation.

In the other 2 men who showed visual field constriction (patients 12 and 19) optic nerve examination remained normal throughout the study, and there were no changes in the cup:disc ratios. Further, there was no change in the photographic appearance of the nerve heads.

\section{CHORIORETINITIS}

Pre-existing chorioretinal changes attributable to onchocerciasis were present in 5 men who were to receive DEC lotion and in 6 who were to receive DEC tablets. In 3 men receiving DEC tablets (patients 3, 5, and 9) and in 4 receiving DEC lotion (patients 1, 2,6, and 10 ) this took the form of peripheral chorioretinal scarring, although in 1 patient (patient 1) this was associated with marked retinal pigment epithelial atrophy in the macular area. One man in the lotion group (patient 16) had bilateral vitritis, bilateral peripheral chorioretinal scars, and epiretinal membranes in the left macular region. A posterior polar chorioretinal scar was present in 1 patient receiving DEC tablets (patient 4), peripapillary atrophy occurred bilaterally in another receiving DEC tablets (patient 19), and a glial proliferation was present in a third (patient 15).

During this study new chorioretinal lesions developed in a total of 5 men. A focal area of acute chorioretinitis was observed at 2 months in 1 man receiving DEC tablets (patient 3) (Fig. 4). This had

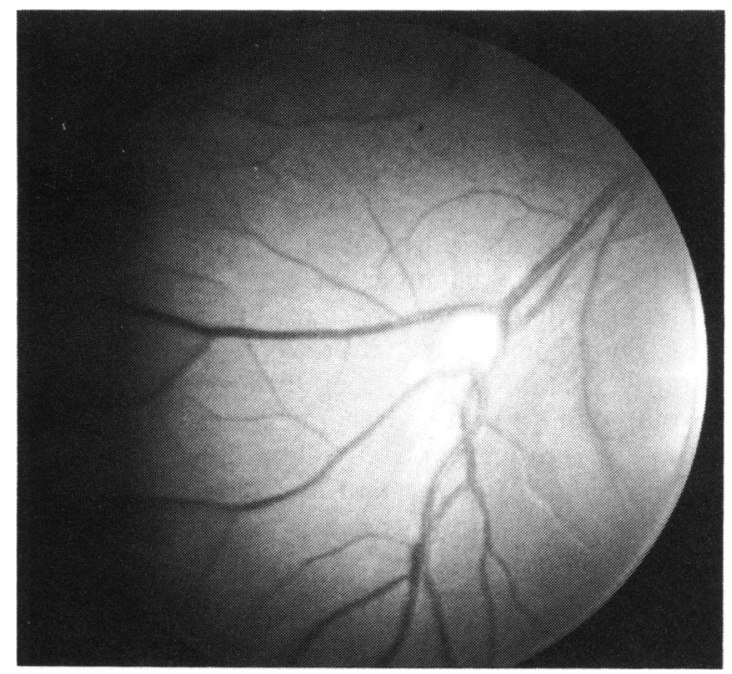

Fig. 4 A focal area of active chorioretinitis observed in a man who had been treated with oral DEC for 2 months (patient 3). progressed to a chorioretinal scar at 6 months. New areas of chorioretinal scarring developed in 4 men receiving lotion (patients $6,8,10$, and 17 ). Two of the men who developed these changes ( 8 and 17) had normal fundal examinations before treatment, and the new lesions were their first evidence of posterior segment disease. The other 3 men had pre-existing chorioretinal lesions. The patient with pre-existing vitritis (patient 16) developed moderately dense vitreous opacities during treatment.

\section{INTRAOCULAR PRESSURE}

The mean intraocular pressure for each group showed no significant change during the course of treatment. Further, no significant rise or fall in pressure was observed in any individual in either group.

\section{Discussion}

The ocular changes seen with the DEC treatment of onchocerciasis have been well described. ${ }^{8}$ They include a dramatic increase in the number of intracorneal microfilariae and the appearance of punctate keratitis (fluffy opacities), which are seen when the microfilariae in the cornea die. These opacities, which clear over a period of time, are formed by the acute inflammatory infiltrate which surrounds dead and dying microfilariae. ${ }^{9} \mathrm{~A}$ marked increase in the number of microfilariae in the anterior chamber with the initiation of treatment and the development or exacerbation of anterior uveitis are also well recognised. Thus, one effect of DEC therapy on microfilariae seems to be to activate or mobilise them, which results in microfilariae being 'driven' into the eye. With time, however, there is usually a reduction in the number of microfilariae in the eye, and there is a temporary improvement of lesions in the anterior segment. There appears to be little change, however, in the progression of posterior lesions. ${ }^{8}$

The conclusions drawn from any clinical trial must be considered in the context of the methodology employed. This study was double-masked, so that neither the investigators nor the patients were aware of which treatment a particular patient was receiving. The code was not broken until all clinical and laboratory studies were completed. The randomisation used in this study produced 2 groups of men that were highly comparable before the start of treatment. Not only were the microfilarial counts similar in each group, but the ocular examination in terms of sclerosing keratitis, anterior uveitis, and chorioretinal changes was also comparable.

The presence of intraocular microfilariae is strongly correlated with the severity of onchocercal lesions and the risk of developing blindness. ${ }^{10}$ It is note- 
worthy in this regard that intraocular microfilariae were not present in either group before treatment. Although microfilariae appeared in both the cornea and the anterior chamber of members of both groups. the number of people with intraocular microfilariae, the time microfilariae stayed in the eye, and the absolute counts of microfilariae, both in the cornea and the anterior chamber, were higher in those receiving DEC lotion than in those receiving DEC tablets. In particular, at 2 and 6 months microfilariae were still present in the eyes of more than half the patients receiving DEC lotion.

Although microfilariae appeared in both the anterior chamber and the cornea during DEC lotion therapy, the greatest migration was into the anterior chamber. Another study, examining the effect of the DEC lotion on intraocular microfilariae, showed an apparent reduction in intracameral microfilarial counts in people receiving the lotion. ${ }^{11}$ As the authors point out, the ophthalmic examinations in this previous study were conducted by a number of different observers, and therefore their findings relating to intraocular microfilariae must be regarded with circumspection. ${ }^{11}$ The present study, however. shows quite clearly that DEC lotion 'drives' microfilariae into the eye, especially into the anterior chamber, to an even greater extent than does oral DEC.

Evidence of optic nerve involvement, that is, visual field constriction and/or optic nerve pallor (optic atrophy), occurred with approximately equal frequency in each group. The generalised constriction of visual fields to 5 to 10 degrees has been described as a common finding in patients with onchocerciasis, and visual acuity usually is not reduced. ${ }^{12}$ Similar visual field defects also have been reported as occurring acutely during oral DEC treatment. ${ }^{13}$

Optic atrophy has long been viewed as part of the clinical picture of onchocerciasis. Recently, however. a number of reports have suggested an association between optic atrophy and the use of onchocerciasis chemotherapy..$^{1314}$ In a study in the Cameroons Anderson and co-workers found that 3 of 18 people treated with oral DEC developed optic atrophy, and 6 of another 21 people showed some changes in the appearance of the optic nerve during DEC treatment. ${ }^{8}$ The risk of optic atrophy was similar in another study in which combined therapy, consisting of DEC followed by suramin, was used. ${ }^{15}$ Using fluorescein angiography Bird and co-workers ${ }^{13}$ found acute optic neuritis in 9 of 11 people receiving oral DEC and an aggravation of fluorescein leakage in 4 of a further 10 patients who had pre-existing leakage. They also demonstrated the frequent development of acute self-limiting lesions of the retinal pigment epithelium. Both the optic nerve changes and the retinal pigment epithelial changes could be recognised only by fluorescein angiography. Neither of these could be observed during the present study because fluorescein angiography was not used. Conversely, optic atrophy developed in 3 patients in our study but is not considered in the study of Bird and co-workers, as their follow-up was for only 8 days. Of particular concern in the present study is the fact that visual field constriction and optic atrophy developed as commonly in people who received DEC lotion as in those who received tablets.

The transepidermal application of DEC as a lotion has been advocated as a way of eliminating systemic absorption, thus preventing ocular and systemic reactions. ${ }^{56}$ Serum levels of DEC in patients receiving DEC lotion are less than one-tenth of those found in patients receiving DEC tablets. ${ }^{3}$ However, sufficient drug appears to be absorbed to initiate the complex and as yet undefined series of events that have plagued the clinical use of oral diethylcarbamazine in onchocerciasis, resulting in both systemic and ocular side effects, including optic atrophy.

The death of microfilariae in the cornea and the development of fluffy corneal opacities are direct evidence that microfilariae are dying inside the eye. This strongly suggests that DEC lotion kills intraocular microfilariae, and indicates that the effect of DEC lotion is not limited to the skin. However, it must be acknowledged that there is the remote possibility that microfilariae lethally damaged by DEC could migrate into the cornea and subsequently die.

An increase in the number of people with anterior uveitis was noticed in both groups. For the most part the anterior uveitis was relatively mild. However, uveitis developed more frequently and was more persistent in those who received DEC lotion. The pathogenesis of uveitis is not clear, but it is possible that it is related to the presence of circulating immune complexes which could cause vasculitis and subsequent leakage of serum proteins and cells from vessels into the anterior chamber. It should be noted that proteinuria has been reported as developing with DEC therapy and is seen more commonly with DEC lotion. ${ }^{16}$ It is interesting, therefore, to note that the development of proteinuria appears to be correlated with the presence of circulating immune complexes. ${ }^{17}$ and it may be that a similar mechanism is involved in causing uveitis.

The development of an area of acute focal chorioretinitis in a patient receiving DEC tablets was especially interesting, as we are not aware of a similar lesion having been previously reported as a result of DEC treatment. It is impossible to tell whether this lesion occurred because of, or in spite of, the concurrent use of DEC. This active lesion developed in 
an eye which already had evidence of onchocercal chorioretinitis, as did the new lesions in 2 patients receiving DEC lotion. It should be noted, however, that new chorioretinal lesions developed in another 2 people who were receiving DEC lotion and who did not have pre-existing chorioretinal lesions. While these probably were not due to a direct effect of the DEC lotion, the use of the lotion certainly did not prevent their appearance.

Several authors have commented on an apparent association of onchocerciasis and glaucoma, particularly juvenile glaucoma, in certain areas of Africa. ${ }^{18} 19$ This led to speculation of a causal relationship of onchocerciasis to glaucoma and to the suggestion that intraocular pressure was lowered by DEC treatment. ${ }^{5}$ More recently, an extensive study has shown that people with onchocerciasis have, if anything, lower intraocular pressures than people without onchocerciasis, except for the few people who develop secondary glaucoma following severe uveitis. ${ }^{20}$ No one in the present study had a raised intraocular pressure, and there was no significant decrease in pressure over time. These findings would support the contention that onchocerciasis per se does not elevate intraocular pressure.

In conclusion, during this study all of the major ocular side effects described as occurring with oral DEC were seen with both topical and oral DEC therapy, with the exception of globular limbitis. The more subtle changes in the retinal pigment epithelium and optic nerve were not seen in this study and could not have been detected without fluorescein angiography. The transepidermal administration of DEC as a $2 \%$ lotion was associated with a higher incidence of ocular changes than was the oral administration of DEC. In particular, those patients receiving DEC lotion had more microfilariae migrate into the anterior chamber and cornea. Patients receiving DEC lotion also had more new chorioretinal changes develop during treatment. Of perhaps even greater significance was the fact that visual field constriction and optic nerve pallor occurred in patients in both groups.

In terms of safety and efficacy there seems to be no reason to prefer DEC lotion to DEC tablets, since previous reports have shown that DEC lotion is only marginally better than placebo lotion ${ }^{11}$ and far less effective than DEC tablets ${ }^{3}$ in reducing microfilarial skin-snip counts. DEC lotion also produces at least as many systemic side effects as DEC tablets. Therefore, from the present study there seems to be no reason for contemplating the use of DEC lotion to reduce the ocular involvement in patients with onchocerciasis on the grounds that the lotion has less 'toxic' side effects, or that it protects the eye by encouraging or allowing the microfilariae to migrate from the eye to other tissues. ${ }^{6}$ In fact the use of the lotion caused the appearance of increased numbers of intraocular microfilariae and was attended by even more ocular changes than seen with oral DEC.

We thank the Firestone Plantations Company, Harbel, Liberia (under the direction of Mr R. C. Larson), Drs Z. D. Traub and E. Jallah, Mary and Willem Kraaij, Jennifer Dennis, Carol Vispo, and other personnel of the Firestone Medical Center and Plantations Company, and Drs M. Del Monte and M. E. Langham, of the Johns Hopkins University, for their co-operation and support.

This work was supported by research grants from the Rockefeller Foundation, New York, and from the Firestone Tire and Rubber Company, Akron, Ohio, and by NIH grants BRS 16-80 and EY-03318.

\section{References}

1 Anderson J, Fuglsang $\mathrm{H}$, Marshall TF. Effects of suramin on ocular onchocerciasis. Tropenmed Parasitol 1976; 27: 279-96.

2 Hawking F. Diethylcarbamazine and new compounds for the treatment of filariasis. Adv Pharmacol Chemother 1979; 16: 129-94.

3 Taylor HR. Greene BM, Langham ME. Controlled clinical trial of oral and topical diethylcarbamazine in treatment of onchocerciasis. Lancet 1980; i: 943-6.

4 Mazzotti L. Possibilidad de utilizar como medio diagnostico auxiliar en la oncocercosis, las reacciones alergicas consecutivas a la administracion de 'Hetrazan'. Rev Inst Salubr Enferm Trop (Mex) 1948; 9: 235-7.

5 Langham ME, Traub ZD, Richardson R. A transepidermal chemotherapy of onchocerciasis. Tropenmed Parasitol 1978; 29: $156-62$.

6 Langham ME. Diethylcarbamazine lotion in onchocerciasis. Lancet 1980; i: 977-8.

7 Jones BR, Anderson J, Fuglsang H. Effects of various concentrations of diethylcarbamazine citrate applied as eye drops in ocular onchocerciasis, and the possibilities of improved therapy from continuous non-pulsed delivery. Br J Ophthalmol 1978; 62: 428-39.

8 Anderson J, Fuglsang H, Marshall TF. Effects of diethylcarbamazine on ocular onchocerciasis. Tropenmed Parasitol 1976; 27: 263-78.

9 Garner A. Pathology of ocular onchocerciasis: human and experimental. Trans R Soc Trop Med Hyg 1976; 70: 374-7.

10 Thylefors B, Brinkmann UK. The microfilarial load in the anterior segment of the eye. A parameter of intensity of onchocerciasis. Bull WHO 1977; 55: 731-7.

11 Taylor HR, Langham ME, de Stahl EM, Figueroa LN, Beltranena F. Chemotherapy of onchocerciasis: a controlled clinical trial of topical diethylcarbamazine (DEC) in Guatemala. Tropenmed Parasitol 1980; 31: 357-64.

12 Thylefors B. Tonjum AM. Visual field defects in onchocerciasis. Br J Ophthalmol 1978; 62: 462-7.

13 Bird AC, El-Sheikh H, Anderson J, Fuglsang H. Changes in visual function and in the posterior segment of the eye during treatment of onchocerciasis with diethylcarbamazine citrate. $\mathrm{BrJ}$ Ophthalmol 1980; 64: 191-200.

14 Thylefors B, Rolland A. The risk of optic atrophy following suramin treatment of ocular onchocerciasis. Bull WHO 1979; 57: 479-80.

15 Anderson $J$, Fuglsang $H$. Further studies on the treatment of ocular onchocerciasis with diethylcarbamazine and suramin. $\mathrm{BrJ}$ Ophthalmol 1978: 62: 450-7.

16 Greene BM, Taylor HR, Humphrey RL. Proteinuria associated with diethylcarbamazine treatment of onchocerciasis. Lancet 1980; i: 254-5.

17 Greene BM, Taylor HR, Humphrey RL, Lawley TJ. Circulating 
immune complexes in onchocerciasis: significance and influence of diethylcarbamazine therapy. Clin Res 1980; 28: 370A.

18 Berghout E. Onchocerciasis and glaucoma in the forest area of Ghana. Trop Geogr Med 1973; 25: 233-7.

19 Langham ME, Frentzel-Beyme RR, Traub Z-D. Intraocular pressure and onchocerciasis infection in Liberia. Ophthalmic Res 1975; $7: 368-80$.

20 Thylefors B. Duppenthaler JL. Epidemiological aspects of intraocular pressure in an onchocerciasis endemic area. Bull WHO 1979; 57: 963-9. 